\title{
КАЛИБРОВКА ТЕПЛОФИЗИЧЕСКИХ МОДЕЛЕЙ ДЛЯ РАСЧЕТА ПАРАМЕТРОВ ЛЕДОПОРОДНЫХ ОГРАЖДЕНИЙ ПРИ НАЛИЧИИ ПОГРЕШНОСТЕЙ В ИСХОДНЫХ ДАННЫХ
}

\author{
А.В. Пугин \\ Горный институт УрО РАН, г. Пермь
}

\begin{abstract}
Аннотация: Статья носит методологический характер и дает понимание отдельных вопросов калибровки теплофизических моделей, связанных с наличием погрешностей в исходных данных. Разработанные и откалиброванные теплофизические модели применяются для численного математического расчета параметров ледопородных ограждений при строительстве горных выработок. На примере показана принципиальная возможность удовлетворительной реконструкции поля температур после калибровки модели ограниченным числом параметров. Множество допустимых решений обратной задачи Стефана при калибровке модели может быть построено путем рационального согласования статистических оценок для невязок вычисленных и измеренных температур.

Ключевые слова: искусственное замораживание пород, ледопородное ограждение, теплофизическая модель, погрешности, калибровка, обратная задача Стефана.
\end{abstract}

Разработка теплофизических моделей является неотъемлемым этапом процесса теплофизических исследований при термометрическом контроле состояния ледопородных ограждений (ЛПО) в процессе строительства шахтных стволов. В каждом конкретном случае теплофизическая модель должна достаточно адекватно описывать тепломассообмен в массиве горных пород, подвергнутом искусственному замораживанию, чтобы предоставить возможность достоверной оценки физического состояния этой многофазной среды [6].

Общая методология построения теплофизических моделей частично описана в литературе [4]. В настоящее время за редким исключением расчеты состояния ЛПО производятся численными методами, что предъявляет определенные требования к систематизации исходной информации в составе модели.

Теплофизическая модель состоит из двух частей: 1) параметрической и 2) математической. В математическую компоненту заложены основные уравнения и граничные условия, определяющие законы протекания термодинамических процессов в сложной системе «замораживающие колонки - массив горных пород - строящийся ствол». Параметрическая составляющая модели содержит формализованную информацию о свойствах среды, естественных и технологических факторах, влияющих на тепломассообмен, и представляет собой конечное упорядоченное множество значений исходных параметров. В параметрическую составляющую модели закладывается вся неопределенность имеющейся исходной информации, поскольку каждый входной параметр измерен приближенно.

За температурное состояние массива горных пород в процессе искусственного замораживания и размораживания отвечает более 15 параметров. В процессе калибровки большинство из них фиксируются, а незначительная часть переопределяется до наилучшего совпадения модельных (вычисленных) и измеренных температур в точках замеров с координатами в контрольно-термических (КТ) скважинах и шпурах. Обычно переопределению подлежат значения теплопроводности мерзлой и незамороженной породы и количество воды, замерзающей в процессе фазового перехода.

Отталкиваясь от первоначальной параметризации модели редко удается получить модельное распределение температур, удовлетворяющее фактическим значениям. Характеристики горных пород, внесенные в модель, определены в лабораторных условиях на образцах керна («точечные» характеристики) и могут служить лишь первым при- 
ближением к удельным характеристикам объемов пород, вовлекаемых в процесс замораживания в естественном залегании [3].

Реконструкция поля температур неразрывно связана с численным решением обратной задачи Стефана с движущейся границей фазового перехода относительно набора нескольких параметров. Единственного решения обратной задачи, обеспечивающего совпадение измеренного и модельного полей с точностью до нуля, на практике добиться невозможно в силу наличия помех и погрешностей в исходных данных. Имеется в общем случае бесконечное, а в каждом конкретном случае - конечное множество частных приближенных решений обратной задачи Стефана, которые будут удовлетворять требуемой невязке модельного и измеренного полей. Существование множества решений обратной задачи, отвечающих одному аномальному полю из-за наличия помех в исходных данных, называют практической эквивалентностью [2].

Поскольку переопределению при калибровке модели подвергается ограниченный набор параметров, возникает закономерный вопрос: возможно ли найти удовлетворительное решение и восстановить распределение температур с приемлемой точностью, калибруя модель ограниченным набором параметров, в которые неизбежно будут заложены все погрешности исходных данных?

Проанализируем ситуацию на примере скрытых погрешностей в данных инклинометрии замораживающих и КТ скважин. В качестве источников поля температур, отбирающих теплоту из массива, будем рассматривать 36 замораживающих скважин (колонок), расположенных в плане по окружности, радиусом 7 м. Расстояние между соседними замораживающими колонками составляет 1,22 м. Моделирование выполнялось для слоя песка с параметрами, близкими к условиям одного из строящихся рудников (табл. 1). Под температурами ликвидуса и солидуса понимаются температуры начала и полного замерзания воды в породе.

Таблица 1

Параметры слоя породы, принятые к расчету

\begin{tabular}{|c|c|c|c|c|c|c|c|c|c|c|c|}
\hline \multirow{2}{*}{ 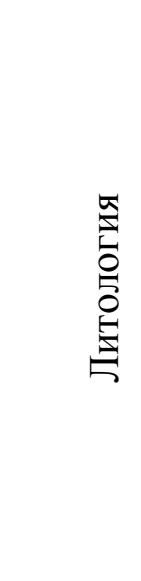 } & \multicolumn{2}{|c|}{ Глубина, м } & \multicolumn{3}{|c|}{ Температура, ${ }^{0} \mathrm{C}$} & \multicolumn{2}{|c|}{$\begin{array}{l}\text { Теплопро- } \\
\text { водность, } \\
\left.\text { Вт/( }{ }^{2} \cdot{ }^{0} \mathrm{C}\right)\end{array}$} & \multicolumn{2}{|c|}{$\begin{array}{c}\text { Удельная } \\
\text { теплоемкость, } \\
\left.\text { Дж/(кг. }{ }^{\circ} \mathrm{C}\right)\end{array}$} & \multirow{2}{*}{ 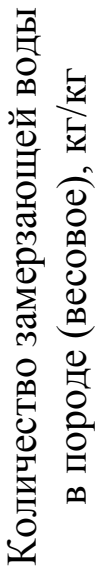 } & \multirow{2}{*}{ 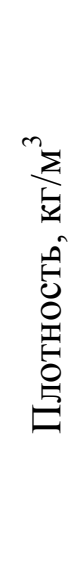 } \\
\hline & 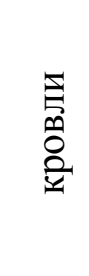 & $\begin{array}{l}\text { 畣 } \\
\text { 言 } \\
\text { 댑 }\end{array}$ & 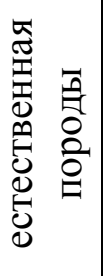 & 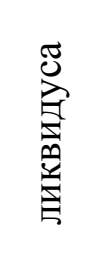 & 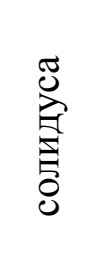 & 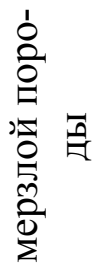 & 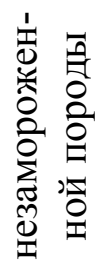 & 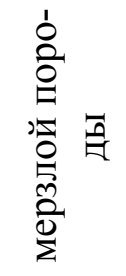 & 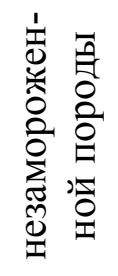 & & \\
\hline $\begin{array}{l}\text { Песок } \\
\text { глини- } \\
\text { стый }\end{array}$ & 120 & 140 & 9,5 & $-0,2$ & $-1,0$ & 3,5 & 2,5 & 922 & 1183 & 0,15 & 2110 \\
\hline
\end{tabular}

Вблизи контура замораживающих колонок пробурены четыре КТ скважины: внешние КТ1 на расстоянии 1,22 м от двух соседних замораживающих скважин, КТ2 на расстоянии 2 м и КТ3 на расстоянии 5 м от окружности замораживающих 
колонок; внутренняя КТ4 на расстоянии 1,22 м от соседних замораживающих колонок напротив КТ1. Первоначально принимается, что все скважины пробурены вертикально.

Модельное поле температур для слоя песка вычислено на конец периода активного замораживания в 120 суток при рабочей температуре прямого потока хладоносителя $-30^{\circ} \mathrm{C}$ и расходе $250 \mathrm{~m}^{3} /$ ч. Этот период включает выход замораживающей станции на рабочий режим с понижением температуры хладоносителя на $3{ }^{0} \mathrm{C} /$ сутки, в качестве которого принимался $29 \%$ водный раствор $\mathrm{CaCl}_{2}$. Численное моделирование выполнялось в 2D постановке в программе «FrozenWall» [5]. Вычисленные в точках КТ скважин значения температур приняты за измеренные для последующего эксперимента.

Далее в положения всех скважин внесена погрешность инклинометрии, полученная с применением генератора псевдослучайных чисел, имеющих нормальное распределение. Минимальное отклонение скважин от вертикали составило 0,04 м, максимальное 0,41 м при математическом ожидании 0,19 м и стандартном отклонении $\pm 0,22$ м. Новые позиции скважин были внесены в модель взамен предыдущих и в процессе ее калибровки не изменялись (рис. 1).

Вычислительный эксперимент заключался в том, чтобы путем варьирования трех параметров, теплопроводностей мерзлой и незамороженной породы и количества замерзающей воды, подобрать распределение температур в слое, наилучшим образом приближающее измеренное поле.

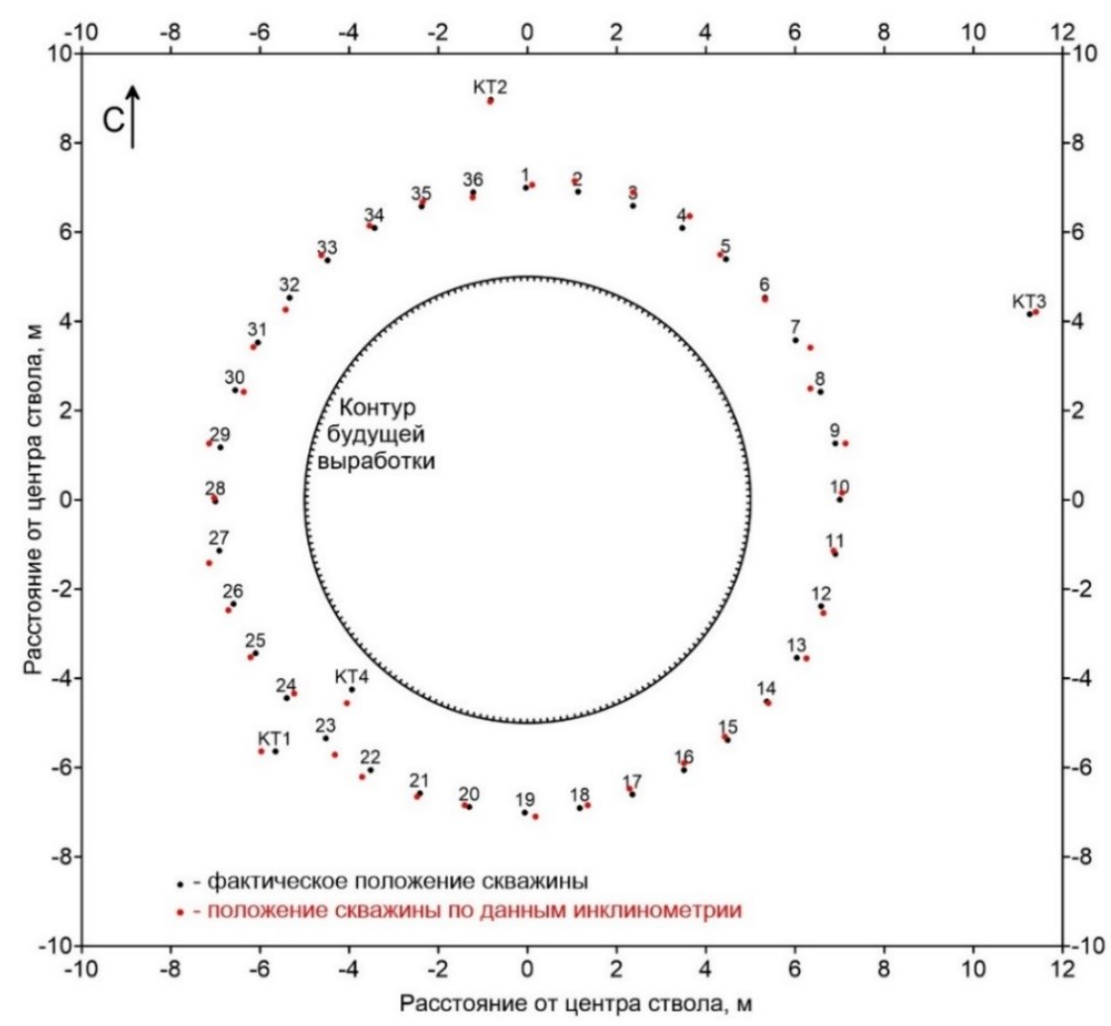

Рис. 1. Положение замораживающих и КТ скважин

Для сопоставления результатов эксперимента с измеренным полем необходимо определиться с метриками, в которых будет вычисляться расстояние между функциями. Обычно для статистической оценки близости двух полей в геофизике применяется стандартное или среднеквадратическое отклонение (метрика $L_{2}$ ) 


$$
S D= \pm \sqrt{\frac{\sum_{i=1}^{N}\left(T_{i}-T_{i}^{*}\right)^{2}}{N}},
$$

где $T_{i}$ - измеренное значение температуры; $T_{i}^{*}$ - модельное значение температуры; $N-$ количество КТ скважин. Минимизация функционала $S D\left[T-T^{*}\right]$ является основным, но недостаточным критерием для определения наилучшего решения.

Логично предположить, что результат будет близок к наилучшему, если отклонения температур по всем КТ скважинам будут центрированы. В этом случае в качестве второго критерия можно принять метрику $L_{1}$ и оценивать близость к нулю среднего арифметического значения $\overline{\delta T}$ отклонений температур (второй, дополнительный критерий). Исходя из опыта автора целесообразно также придерживаться двух других дополнительных критериев, совместно обеспечивающих центрирование расхождений:

1) число отклонений одного знака должно быть равно числу отклонений другого знака в предположении о приближенном равенстве нулю медианного значения отклонений, другими словами, $M e\left[T-T^{*}\right] \approx 0-$ третий критерий [1];

2) наименьшее отрицательное и наибольшее положительное отклонения должны быть близки по модулю, то есть предельное отклонение одного знака не должно быть существенно больше предельного отклонения другого знака, что можно проконтролировать, приближая к нулю среднее диапазонное значение $M R\left[T-T^{*}\right]=$ $\frac{\min (\delta T)+\max (\delta T)}{2}-$ четвертый критерий (MR от англ. «Midrange»).

Представляется, что в совокупности эти статистики обеспечат нахождение компактного множества допустимых решений обратной задачи Стефана. Указанные критерии не будет лишним дополнить оценкой в метрике Чебышева, т. е. контролировать максимальное по модулю отклонение температур, либо минимаксной оценкой - контроль максимальных по модулю отклонений обоих знаков.

В результате серии численных решений прямой задачи с полным перебором трех калибруемых параметров (табл. 2) вычислены значения всех статистик для отклонений модельных и измеренных температур.

Таблица 2

Диапазоны и шаг изменения калибруемых параметров

\begin{tabular}{|c|c|c|c|c|c|c|c|c|}
\hline \multicolumn{3}{|c|}{$\begin{array}{c}K_{1} \text { - теплопроводность не- } \\
\text { замороженной породы, } \\
\text { Вт } /\left(\mathrm{M}^{2} \cdot{ }^{0} \mathrm{C}\right)\end{array}$} & \multicolumn{3}{|c|}{$\begin{array}{c}K_{2} \text { - теплопроводность } \\
\text { мерзлой породы, } \\
\text { Вт } /\left(\mathrm{m}^{2}{ }^{0} \mathrm{C}\right)\end{array}$} & \multicolumn{3}{|c|}{$\begin{array}{c}W \text { - количество замерзаю- } \\
\text { щей воды в породе (весо- } \\
\text { вое), кг/кг }\end{array}$} \\
\hline Мин. & Макс. & Шаг & Мин. & Макс. & Шаг & Мин. & Макс. & Шаг \\
\hline 1,5 & 3,5 & 0,5 & 2,5 & 4,5 & 0,5 & 0,1 & 0,2 & 0,025 \\
\hline
\end{tabular}

Множество допустимых решений обратной задачи Стефана может быть построено путем нахождения области пересечения всех функционалов невязки в пределах выбранных пороговых значений. В данном случае для определения области пересечения использовались следующие пороговые значения: $S D \leq \pm 1,4{ }^{\circ} \mathrm{C} ;|\overline{\delta T}| \leq 0,5^{\circ} \mathrm{C} ; \mathrm{Me} \leq$ $0,5^{\circ} \mathrm{C} ; M R \leq 0,25^{\circ} \mathrm{C}$. Анализ функционалов выполнялся в плоскостях $K_{1} O K_{2}$ при равных значениях $W$ (рис. 2).

Принципиально все решения в пределах области пересечения могут рассматриваться как равнозначные. Выбор «наилучшего» из них может быть осуществлен по какомулибо критерию, например, по минимальному значению $S D$, либо используя иные оценки [1]. Согласно $S D$ отобрано пять частных решений, по одному в каждой плоскости $W$ $=$ Const (табл. 3) $\mathrm{c}$ шагом по теплопроводностям $0,1 \mathrm{BT} /\left(\mathrm{M}^{2} \cdot{ }^{0} \mathrm{C}\right)$. 
Параметры и статистики выбранных частных решений

Таблица 3

\begin{tabular}{|c|c|c|c|c|c|c|c|c|c|}
\hline № & $\begin{array}{c}K_{1}, \\
\mathrm{BT} /\left(\mathrm{M}^{2} \cdot{ }^{\circ} \mathrm{C}\right)\end{array}$ & $\begin{array}{c}K_{2}, \\
\mathrm{BT} /\left(\mathrm{M}^{2} \cdot{ }^{\circ} \mathrm{C}\right)\end{array}$ & $\begin{array}{c}W, \\
\mathrm{\kappa г} / \mathrm{\kappa} \Gamma\end{array}$ & $S D,{ }^{0} \mathrm{C}$ & $\overline{\delta T},{ }^{\circ} \mathrm{C}$ & $\mathrm{Me},{ }^{0} \mathrm{C}$ & $M R,{ }^{0} \mathrm{C}$ & $\mathrm{Min},{ }^{0} \mathrm{C}$ & $\mathrm{Max},{ }^{0} \mathrm{C}$ \\
\hline 1 & 2,5 & 2,0 & 0,100 & $\pm 1,355$ & 0,093 & $-0,043$ & 0,230 & $-1,489$ & 1,949 \\
\hline 2 & 3,1 & 2,5 & 0,125 & $\pm 1,249$ & $-0,226$ & $-0,461$ & 0,010 & $-1,590$ & 1,609 \\
\hline 3 & 3,6 & 2,6 & 0,150 & $\pm 1,236$ & $-0,325$ & $-0,449$ & $-0,202$ & $-1,734$ & 1,475 \\
\hline 4 & 4,1 & 2,9 & 0,175 & $\pm 1,220$ & $-0,271$ & $-0,494$ & $-0,048$ & $-1,679$ & 1,583 \\
\hline 5 & 4,5 & 3,0 & 0,200 & $\pm 1,230$ & $-0,138$ & $-0,329$ & 0,052 & $-1,653$ & 1,758 \\
\hline
\end{tabular}
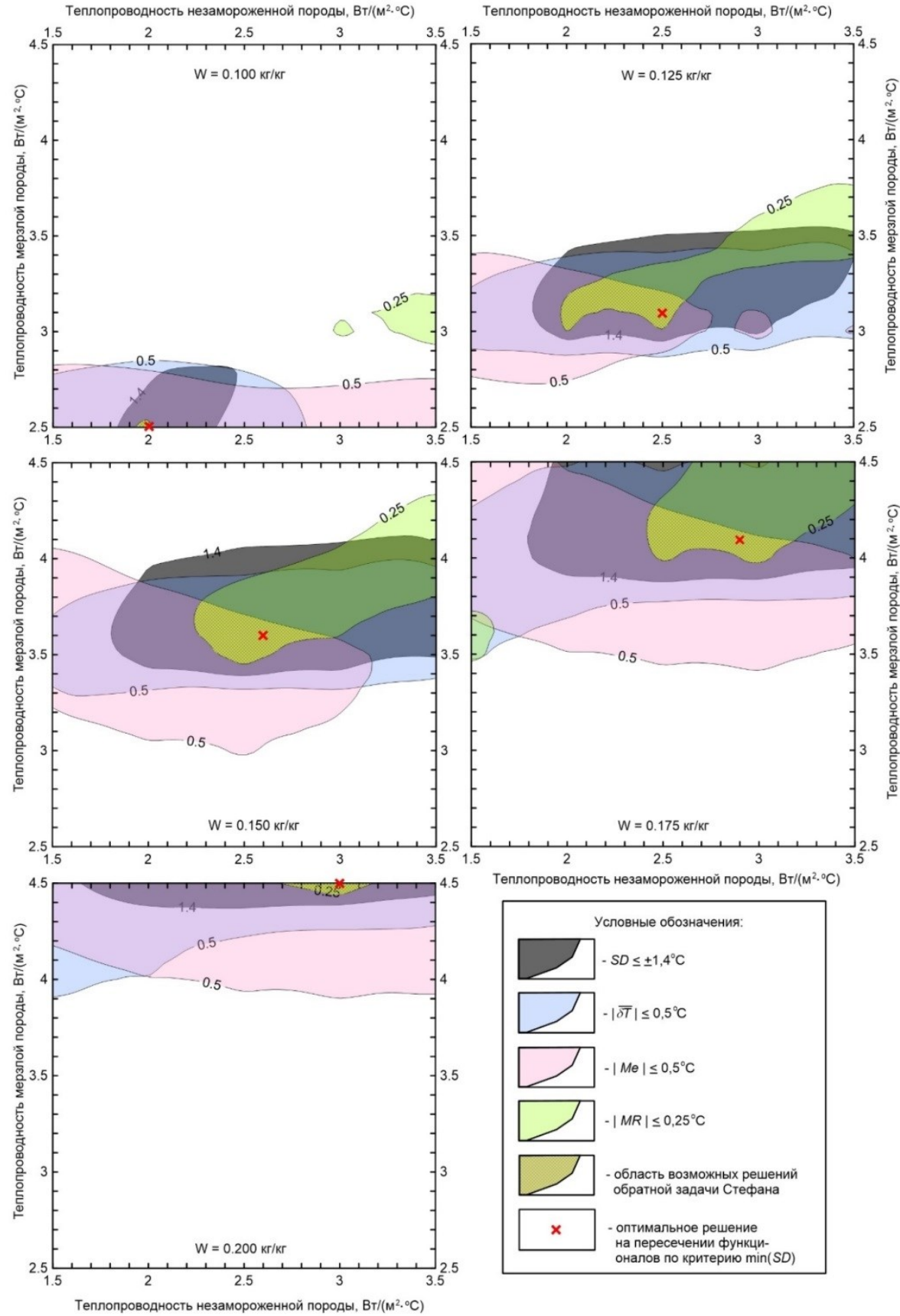

Рис. 2. Согласование функционалов невязки модельных и измеренных температур при заданных пороговых значениях и различном количестве замерзающей воды в породе $\mathrm{W}$ 

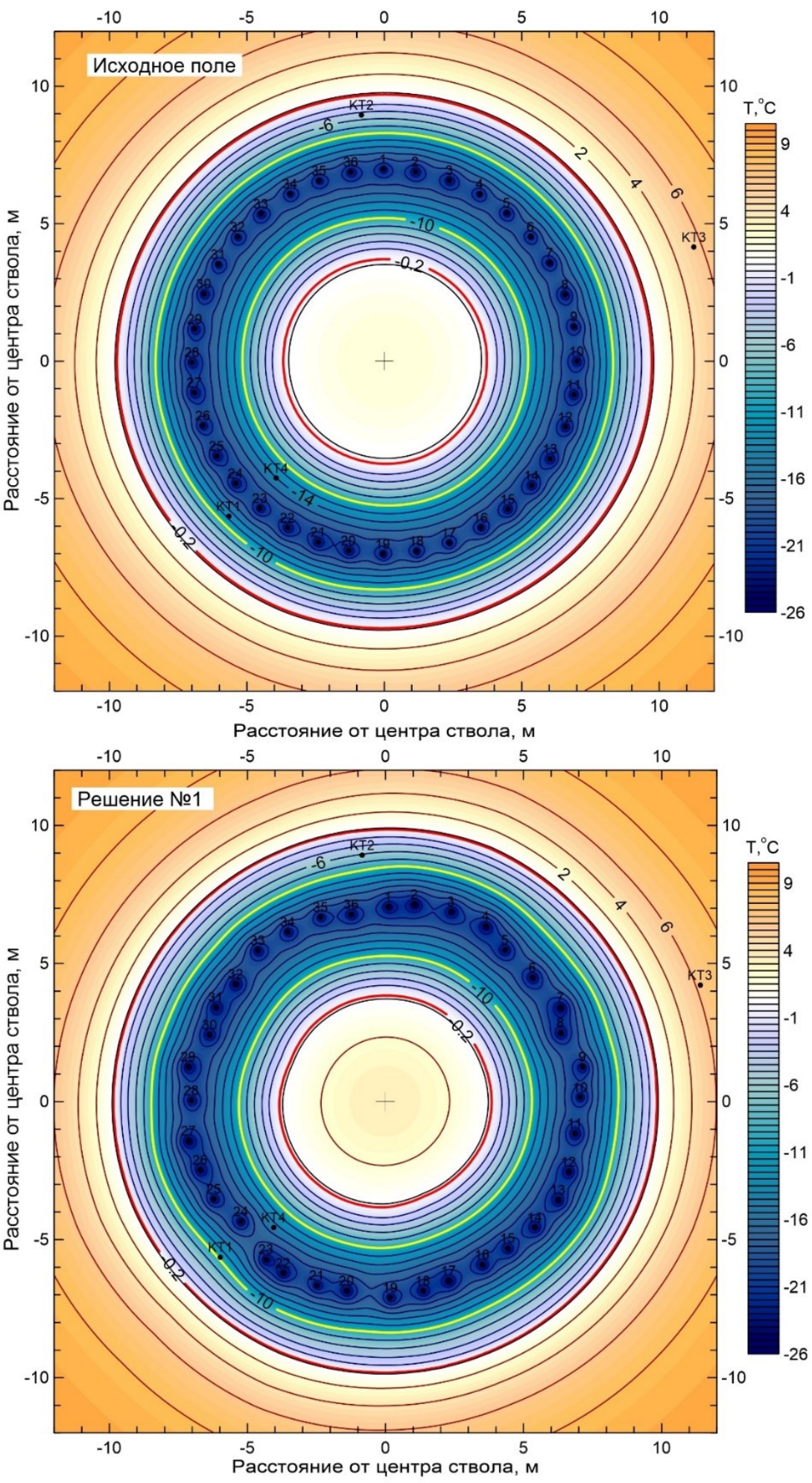

Рис. 3. Распределение температур в слое: исходное (вверху) и для решения № 1 (внизу)

Полученные результаты можно сопоставить и по набору параметров ЛПО, оценка которых обычно производится в процессе термометрического контроля:

1) минимальная толщина ЛПО по температуре ликвидуса;

2) минимальная толщина ЛПО по температуре прочной породы, при которой выполнялись геомеханические исследования образцов керна, например, $-10{ }^{\circ} \mathrm{C}$;

3) средняя температура ЛПО. 
Указанные параметры ЛПО для выбранных решений представлены в табл. 4, где под № 0 указаны параметры ЛПО для исходного варианта поля температур, принятого за эталон.

\section{Параметры ЛПО для выбранных частных решений}

Таблица 4

\begin{tabular}{|c|c|c|c|}
\hline № & $\begin{array}{c}\text { Минимальная толщина } \\
\text { ЛПО по температуре } \\
\text { ликвидуса, м }\end{array}$ & $\begin{array}{c}\text { Минимальная толщина } \\
\text { ЛПО по температуре - } \\
10{ }^{\circ} \mathrm{C}, \mathrm{M}\end{array}$ & $\begin{array}{c}\text { Средняя температура } \\
\text { ЛПО, }{ }^{\circ} \mathrm{C}\end{array}$ \\
\hline 0 & 5,92 & 2,99 & $-10,473$ \\
\hline 1 & 5,92 & 2,96 & $-10,823$ \\
\hline 2 & 6,09 & 3,06 & $-10,757$ \\
\hline 3 & 6,10 & 3,08 & $-10,703$ \\
\hline 4 & 6,12 & 3,04 & $-10,534$ \\
\hline 5 & 6,01 & 2,97 & $-10,410$ \\
\hline
\end{tabular}

Несмотря на значительные максимальные по модулю отклонения и стандартное отклонение выбранных решений от измеренного поля температур, полученные в результате расчета параметры ЛПО всех решений близки между собой и к исходному эталону. Скорее всего это связано с тем, что после длительного периода активного замораживания в 120 суток ЛПО уже имеет значительные размеры и локальные погрешности инклинометрии не оказывают существенного влияния на его глобальное состояние.

В то же время из решения обратных задач геофизики известно, что минимизация функционала невязки или приемлемое совпадение наблюденного и модельного полей в некоторой метрике является «слабым» критерием достоверности полученного результата [1]. Так минимальное значение $S D$ имеет решение № 4, которое одновременно демонстрирует наибольшее отклонение толщин ЛПО от эталона. Тогда как решение № 1 по критерию минимизации $S D$ находится на пятом месте, но дает наиболее близкие к эталону оценки толщины ЛПО, хотя и немного заниженные. С практической точки зрения недооценка лучше переоценки, которая создает дополнительные риски для безопасности. Отметим, что решение № 1 является наихудшим в метрике Чебышева, т. е. согласно максимальному по модулю отклонению температур в $1,949^{\circ} \mathrm{C}$. Исходное распределение температур и полученное в результате данного решения приведены на рис. 3. Визуально в подобранном варианте отмечается искажение формы изоаномал поля температур, вызванное отклонениями скважин от их первоначального положения.

\section{Выводы}

Таким образом, первоначальное исследование с внесением погрешностей инклинометрии показало, что на основании разумного согласования статистических оценок невязок модельных и измеренных температур возможно построить компактное множество допустимых решений обратной задачи Стефана, а также выбрать наилучшие из них с точки зрения реконструкции поля температур и оценки параметров ЛПО.

Откалиброванные параметры модели приобретают характер эквивалентов и могут не отвечать удельным свойствам породы в целом, поскольку призваны частично нивелировать погрешности остальных исходных данных, оставшихся неизменными. В 
статье не исследовалась калибровка теплофизической модели на разные моменты времени, а следовательно, нельзя утверждать о надежности выбранных решений в плане прогноза.

Исследование выполнено при финансовой поддержке Программы ФНИ, проект № 0422-2019-0145-С-01 (регистрационный номер темь НИОКТР: AAAA-A18-118040690029-2).

\title{
БИБЛИОГРАФИЧЕСКИЙ СПИСОК
}

1. Балк П.И., Долгаль А.С., Балк Т.В., Христенко Л.А. Согласование конкурирующих вариантов интерпретации гравитационных аномалий методом минимизации эмпирического риска // Геоинформатика. - 2015. - № 4. - С. 24-35.

2. Блох Ю.И. Интерпретация гравитационных и магнитных аномалий [Электронный ресурс]: учеб. пособие. - 2009. - 232 с. - URL: http://www.sigma3d.com/pdf/books/blokh-2009.pdf.

3. Левин Л.Ю., Семин М.А., Зайцев А.В. Калибровка теплофизических свойств породного массива при моделировании формирования ледопородного ограждения строящихся шахтных стволов // Физикотехнические проблемы разработки полезных ископаемых. - 2019. - № 1. - С. 172-184.

4. Паршаков О.С. Разработка автоматизированной системы термометрического контроля ледопородных ограждений: дис. ...к.т.н.; 25.00.20 / Паршаков Олег Сергеевич. - Пермь, 2020. -140 с.

5. «Frozen Wall»: программа для ЭВМ: свидетельство о гос. регистрации № 2018666337 / Богомягков А.В., Зайцев А.В., Клюкин Ю.А., Левин Л.Ю., Паршаков О.С., Пугин А.В., Семин М.А.; заявитель и правообладатель ПФИЦ УрО РАН. - 2018663501; заявл. 28.11.2018; зарегистрировано 17.12.2018; опубл. 17.12.2018. - 1 c.

6. Левин Л.Ю., Семин М.А., Зайцев А.В. Контроль и прогноз формирования ледопородного ограждения с использованием оптоволоконных технологий // Стратегия и процессы освоения георесурсов: сб. науч. тр. Вып. 14 / ГИ УрО РАН. - Пермь, 2016. - С. 236-238.

\section{ВЗАИМОСВЯЗЬ ФИЗИЧЕСКИХ МЕХАНИЗМОВ, МАТЕМАТИЧЕСКИХ МОДЕЛЕЙ И ТЕХНИЧЕСКИХ СПОСОБОВ ПРОВЕТРИВАНИЯ ТУПИКОВЫХ ГОРНЫХ ВЫРАБОТОК}

\author{
Г.З. Файнбург, М.А. Семин, А.Г. Исаевич \\ Горный институт УрО РАН, г. Пермь
}

\begin{abstract}
Аннотация: Рассматривается взаимосвязь механизмов массопереноса в рудничной атмосфере, математических моделей их аналитического описания и технических способов проветривания тупиковых горных выработок. Методами математического моделирования показано, что реальный процесс проветривания происходит медленнее, чем это описывается моделью идеального смешения, поскольку в структуре воздушных протоков сосуществуют отдельные вихри и застойные зоны с пониженным массообменом с другими потоками.
\end{abstract}

Ключевые слова: идеальное вытеснение, идеальное смешение, математическая модель, тупиковая выработка, способ проветривания.

\section{Введение}

Тупиковые горные выработки являются, с одной стороны, неотьемлемой частью горных работ, а с другой стороны, представляют собой для проветривания гораздо более сложный случай, чем проветривание сквозных выработок. Согласно правилам безопасности [1] в тупиковых забоях длиной более 10 м необходимо осуществлять принудительное проветривание с помощью вентилятора местного проветривания (ВМП), расположенного на сквозной воздушной струе, и вентиляционного трубопровода, про- 